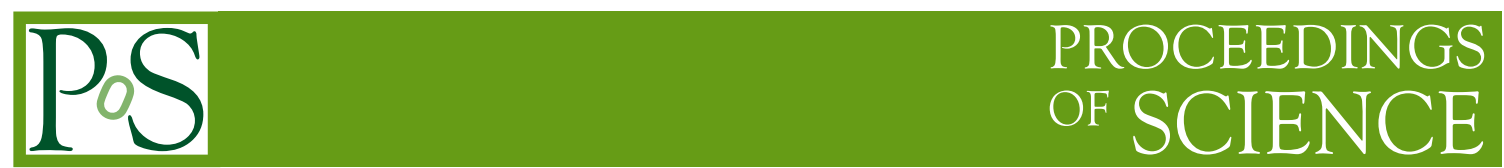

\title{
LHCb Overview
}

\author{
Matthew Kenzie ${ }^{* \dagger}$ \\ CERN \\ E-mail: matthew.kenzie@cern.ch
}

An overview of selected results from the LHCb Collaboration for data collected in Run 1 corresponding to luminsoties of $1 \mathrm{fb}^{-1}$ at $\sqrt{s}=7 \mathrm{TeV}$ and $2 \mathrm{fb}^{-1}$ at $\sqrt{s}=8 \mathrm{TeV}$.

53rd International Winter Meeting on Nuclear Physics,

26-30 January 2015

Bormio, Italy

* Speaker.

† On behalf of the LHCb Collaboration 


\section{Introduction}

The LHCb experiment provides a unique insight into the properties of the Standard Model (SM) and potential New Physics (NP) by analysing $b$ and $c$ mesons and baryons produced in $p-p$ collisions at the LHC. Rare decays which are heavily supressed and precisely predicted in the SM provide excellent candidates as NP can enter the decay diagrams via virtual loops which allow probing energy scales far beyond direct reach at LHC collision energies. NP contributions to these decays can be observed by measuring angular distributions and branching fractions of rare decays. $\mathrm{CP}$ symmetry violation (CPV) is another potential source of NP given that the SM predictions of CPV are far lower than that needed to explain the baryon asummetry in the universe. Precise measurement of the CKM matrix elements, which describes quark mixing in the SM, can provide hints on the scale and impact of NP on the SM. Selected results of LHCb measurements of rare decays and CPV are shown for the Run 1 dataset of $p-p$ collisions at the LHC which consists of data corresponding to $1 \mathrm{fb}^{-1}$ taken at $\sqrt{s}=7 \mathrm{TeV}$ in 2011 and $2 \mathrm{fb}^{-1}$ taken at $\sqrt{s}=8 \mathrm{TeV}$ in 2012.

\section{The LHCb experiment}

The $\mathrm{LHCb}$ detector [1] is a single-arm forward spectrometer covering the pseudorapidity range $2<\eta<5$, designed for the study of particles containing $b$ or $c$ quarks originating from $p-p$ collisions at the LHC. The detector includes a high-precision tracking system consisting of a siliconstrip vertex detector surrounding the $p p$ interaction region, a large-area silicon-strip detector located upstream of a dipole magnet with a bending power of approximately $4 \mathrm{Tm}$, and three stations of silicon-strip detectors and straw drift tubes placed downstream of the magnet. The combined tracking system provides a momentum measurement with relative uncertainty that varies from $0.4 \%$ at $5 \mathrm{GeV} / c$ to $0.6 \%$ at $100 \mathrm{GeV} / c$, and impact parameter resolution of $20 \mu \mathrm{m}$ for tracks with high transverse momentum $\left(p_{\mathrm{T}}\right)$. Charged hadrons are identified using two ring-imaging Cherenkov detectors [2]. Photon, electron and hadron candidates are identified by a calorimeter system consisting of scintillating-pad and preshower detectors, an electromagnetic calorimeter and a hadronic calorimeter. Muons are identified by a system composed of alternating layers of iron and multiwire proportional chambers [3].

A cross-sectional diagram of the detector is shown in Fig. 1 where the proton beams originate from the left and right of the diagram, and the interaction point is inside the vertex locator (VELO). The LHCb detector covers a unique pseduorapidity $(\eta)$ range where most $b$ and $c$ quarks are produced.

The high bunch crossing rate at the LHC ( $\sim 20 \mathrm{MHz}$ during Run 1 data taking) requires a sophisticated trigger system in order to reduce the rate of events which are written to storage $(\sim 5 \mathrm{kHz})$. The trigger [4] consists of a hardware stage, based on information from the calorimeter and muon systems, followed by a software stage, which applies a full event reconstruction. The software trigger requires a two-, three- or four-track secondary vertex with a high sum of the transverse momentum, $p_{\mathrm{T}}$, of the tracks and a significant displacement from the primary $p-p$ interaction vertices (PVs). At least one track should have $p_{\mathrm{T}}>1.7 \mathrm{GeV} / c$ and $\chi_{\mathrm{IP}}^{2}$ with respect to any primary interaction greater than 16 , where $\chi_{\mathrm{IP}}^{2}$ is defined as the difference in $\chi^{2}$ of a given 


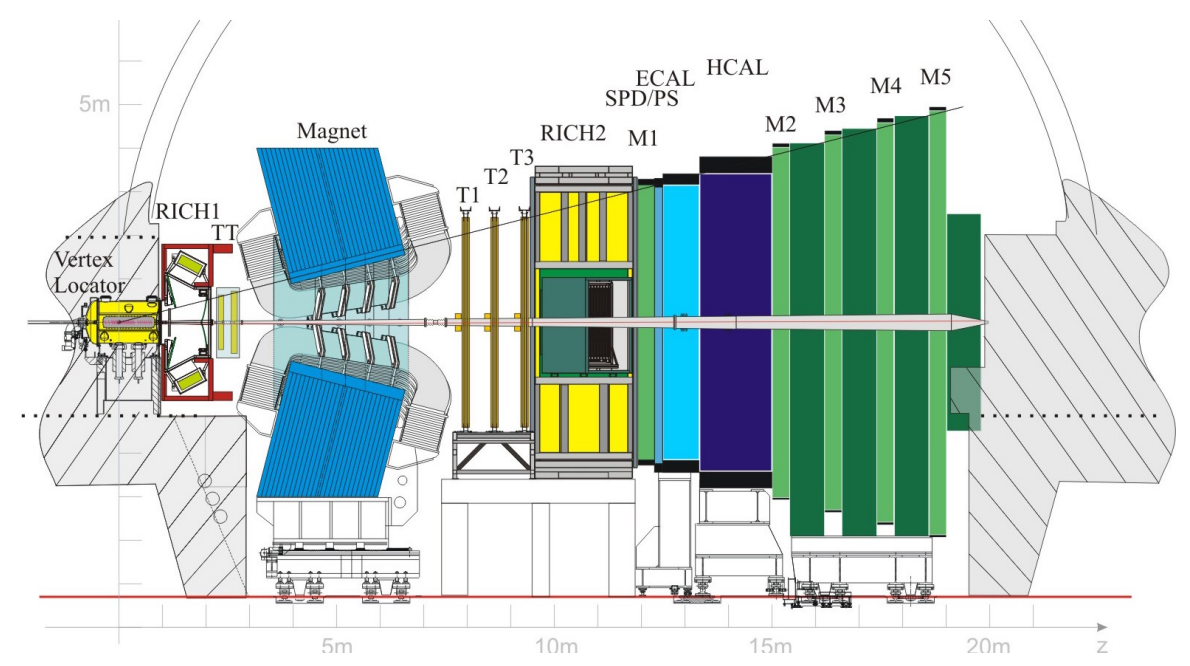

Figure 1: Schematic of the LHCb experiment

PV reconstructed with and without the considered track. A multivariate algorithm is used for the identification of secondary vertices consistent with the decay of a $b$ hadron.

A common analysis technique used at $\mathrm{LHCb}$ is multivariate classifiers usually implemented via a boosted decision tree (BDT) [5] with the AdaBoost algorithm [6] to separate signal from background.

\section{Rare Decays}

Of particular interest at $\mathrm{LHCb}$ are rare decays that are strongly suppressed in the Standard Model (SM), where new physics amplitudes could be sizeable. These include (but are not confined to) the very suppressed decays $B_{d, s}^{0} \rightarrow \mu^{+} \mu^{-}$and electroweak penguin decays of the type $b \rightarrow$ $s \ell^{+} \ell^{-}$which provide stringent constraints on extensions of the SM.

\section{1 $B_{s}^{0} \rightarrow \mu^{+} \mu^{-}$and $B^{0} \rightarrow \mu^{+} \mu^{-}$decays}

The rare, helicity supressed decays $B_{s}^{0} \rightarrow \mu^{+} \mu^{-}$and $B^{0} \rightarrow \mu^{+} \mu^{-}$proceed in the SM by box or penguin-like annihilation diagrams involving the $W^{ \pm}$and $Z$ bosons and the $t$ quark. They are theoretically well understood and their incredibly small predicted branching fractions make them excellent candidates for searching for new physics, up to high energy scales, which can enter the box or annihilation diagrams as virtual particles.

First evidence of the decay $B_{s}^{0} \rightarrow \mu^{+} \mu^{-}$was announced by LHCb in Summer 2012 using $2 \mathrm{fb}^{-1}$ of data from 2011 and 2012 [7]. Recently both the LHCb and CMS collaborations have updated their results to the full Run 1 dataset $[8,9]\left(3 \mathrm{fb}^{-1}\right.$ and $25 \mathrm{fb}^{-1}$ respectively) and a combination of the two results has been submitted to Nature [10]. The analysis techniques of both experiments are similar: a boosted decision tree (BDT) is trained to seperate the dimuon signal from the background. A binning scheme is optimised using the BDT output to group events with similar sensitivty given a variety of input information, such as decay kinematics, detector performance etc. Invariant dimuon mass fits are perfomed simultaneously in each BDT bin using a global 


\begin{tabular}{c|c|c|c|c} 
& SM Prediction & LHCb [8] & CMS [9] & Combination [10] \\
\hline$B_{s}^{0} \rightarrow \mu^{+} \mu^{-}\left(10^{-9}\right)$ & $3.65 \pm 0.23$ & $2.9_{-1.0}^{+1.1+0.3}(4.0 \sigma)$ & $3.0_{-0.9}^{+1.0}(4.3 \sigma)$ & $2.8_{-0.6}^{+0.7}(6.2 \sigma)$ \\
$B^{0} \rightarrow \mu^{+} \mu^{-}\left(10^{-10}\right)$ & $1.06 \pm 0.09$ & $3.7_{-2.1}^{+2.4+0.6}(2.0 \sigma)$ & $3.5_{-1.8}^{+2.1}(2.0 \sigma)$ & $3.9_{-1.4}^{+1.6}(3.2 \sigma)$
\end{tabular}

Table 1: Branching fraction predictions and measurements from $\mathrm{LHCb}$ and CMS with the Run I dataset.

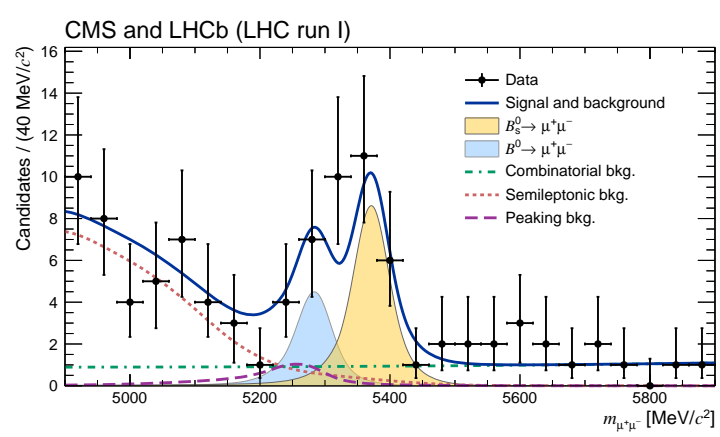

(a)

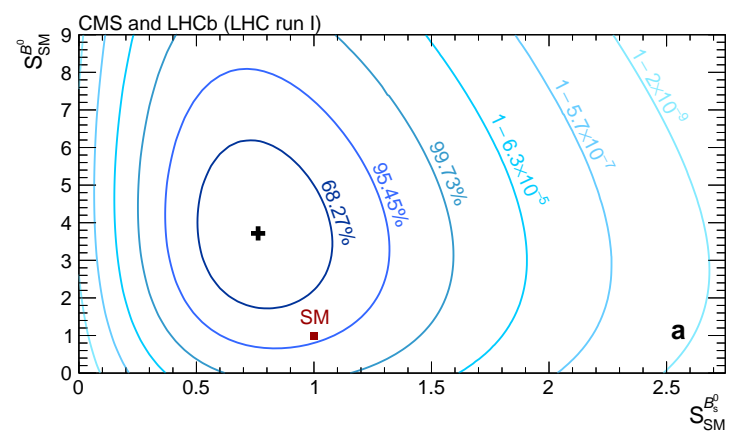

(b)

Figure 2: (Left) The combined dimuon invariant mass spectrum and result of the likelihood fit. (Right) The combined confidence level contours for the $B_{s}^{0} \rightarrow \mu^{+} \mu^{-}$and $B^{0} \rightarrow \mu^{+} \mu^{-}$branching fractions

maximum likelihood approach. The final $B_{s}^{0} \rightarrow \mu^{+} \mu^{-}$yield is normalised to a mode with a well known branching fraction, namely the $B^{+} \rightarrow J / \psi K^{+}$decay. The combination of both experiments observes the $B_{s}^{0} \rightarrow \mu^{+} \mu^{-}$decay over the background with a significance of $6.2 \sigma$ and see evidence for the $B^{0} \rightarrow \mu^{+} \mu^{-}$decay with a significance of $3.2 \sigma$. The predicted and measured branching fractions for the two decays are shown in Table 1. The combined invariant mass fit of the dimuon spectra and the confidence level contours of the decay branching fractions are shown in Fig. 2. The measured $B_{s}^{0} \rightarrow \mu^{+} \mu^{-}$is compatible with the SM prediction whilst the observed $B^{0} \rightarrow \mu^{+} \mu^{-}$BR is a little higher than expected, compatbile with the SM at the $5 \%$ level. More data in Run II and III will determine whether the upward fluctuation in the $B^{0} \rightarrow \mu^{+} \mu^{-}$branching fraction is just a statistical fluctuation or an indication of new physics. The measured branching fractions whilst consistent with the SM provide constraints on many new physics models, particularly those with large $\tan \beta[11,12]$, another demonstration of the importance of this result.

\subsection{Flavour Changing Neutral Current (FCNC) decays $B^{0} \rightarrow K^{* 0} \ell^{+} \ell^{-}$}

The exclusive decays $B^{0} \rightarrow K^{* 0} \mu^{+} \mu^{-}$and $B^{0} \rightarrow K^{* 0} e^{+} e^{-}$proceed in the SM via a penguin or box diagram with a $b \rightarrow s$ transition where the $d$ quark in the $B^{0}$ meson acts as a "spectator" quark. They are sensitive to new physics contributions in the loops and provide a rich set of angular observables with varying sensitivies to new physics. The commonly reconstructed decay angles for four body decays are shown in Fig. 3 (top left). Several theoretical predictions are available and are affected by varying levels of hadronic uncertainties. In some cases the ratio of observables means uncertainties cancel providing a clean test of the SM. Previously LHCb have measured a new set of angular observables [13] suggested in Ref. [14]. This data showed a $3.7 \sigma$ local deviation from the SM in one $q^{2}$ bin of the $P_{5}^{\prime}$ observable. The observable is defined as $P_{5}^{\prime}=S_{5} / \sqrt{F_{L}\left(1-F_{L}\right)}$ where $S_{5}$ is the asymmetry between the red and blue regions of the decay angle plane, shown in Fig. 3 (bottom left) and $F_{L}$ is the longitudinal polarisation fraction of the $K^{* 0}$ resonance. This 

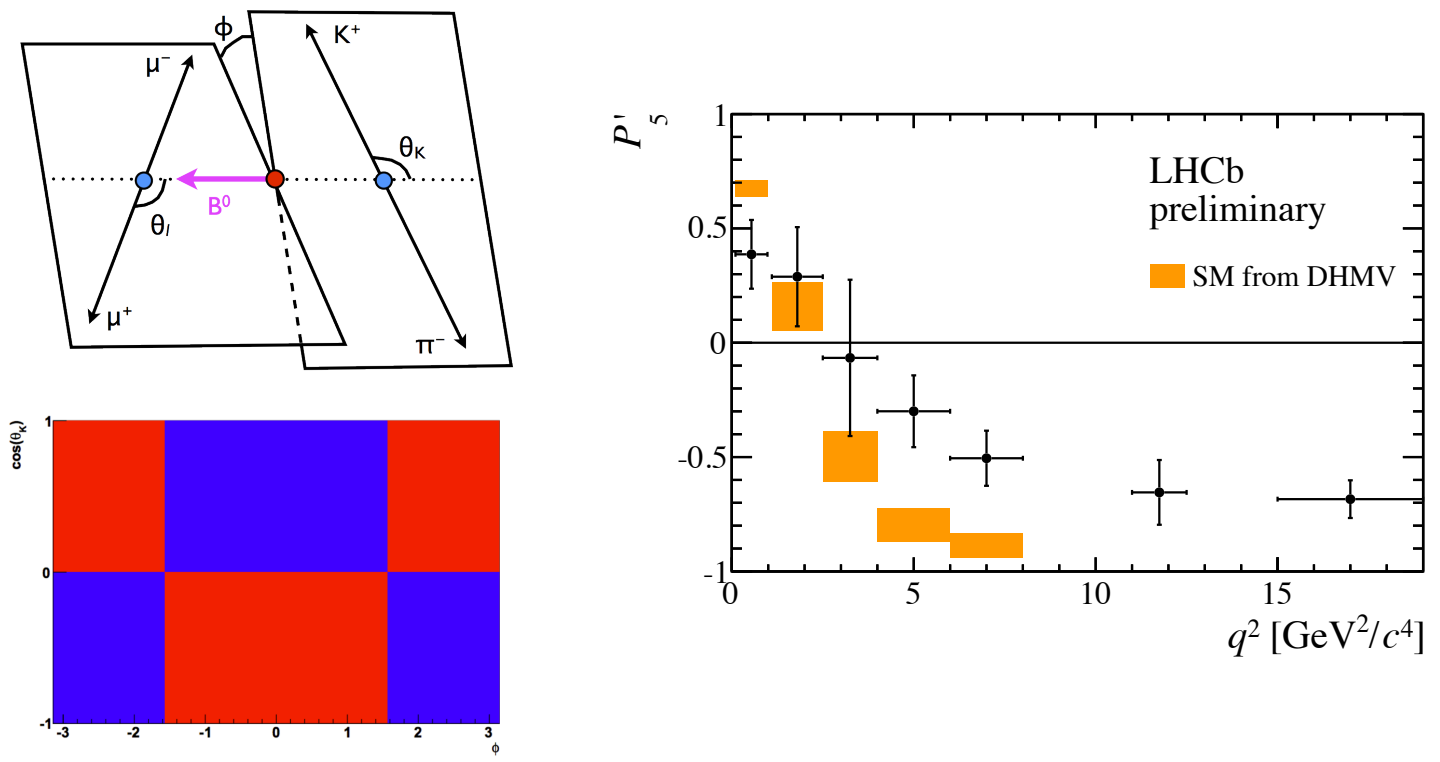

Figure 3: (Top left) Definitions of decay angles. (Bottom left) Definition of the $S_{5}$ angular variable, the asymmetry between the red and blue regions. (Right) Comparison of the LHCb data and the theory prediction from [14] for the $P_{5}^{\prime}$ variable in bins of lepton invariant mass squared, $q^{2}$.

measurement has been recently updated with the full Run 1 dataset and shown at the Moriond conference. The discrepancy is apparent in the 2012 data as well in the $q^{2}$ region between 4 and $8 \mathrm{GeV}$. These results have triggered much interest in the theory community and it remains to be well understood if this fluctuation can be explained by an underestimate of form factor uncertainties or is a hint of new physics. There are some theoretical models which can explain this result and the $P_{5}^{\prime}$ anomaly with very large mass $Z$-like particle. It remains to be seen whether these measurements are cemented with new data or not.

\subsection{Lepton universality in $B^{+} \rightarrow K^{+} \ell^{+} \ell^{-}$}

In the SM the ratio of branching fractions of the $B^{+} \rightarrow K^{+} \mu^{+} \mu^{-}$and $B^{+} \rightarrow K^{+} e^{+} e^{-}$decays, $R_{K}$, is expected to be unity within 1 per mille. The decay diagrams for these are similar to the $B^{0} \rightarrow K^{* 0} \ell^{+} \ell^{-}$decays which proceed in the SM via a penguin or box $b \rightarrow s$ transition instead with a $u$ as the spectator. This is highly sensitive to flavour violating new physics in the loops. The experimental challenge for LHCb is in the electron final state, both in terms of statistics, experimental precision and systematic uncertainties. The ratio of branching fractions $R_{K}$ is computed using the double ratios with the $B^{+} \rightarrow K^{+} J / \psi\left(\rightarrow \ell^{+} \ell^{-}\right)$to cancel the systematic uncertainties, this makes the reasonable assumption of lepton universality for the $J / \psi$. The invariant mass distributions for the two decays are shown in Fig. 4 and the value of $R_{K}$ in comparison to other experiments is shown in Fig. 5. The LHCb measured value of $R_{K}$ is lower than the SM prediction at $R_{K}=0.745_{-0.074}^{+0.090}$ (stat) \pm 0.036 (syst) which is compatible at the $1 \%$ level. 

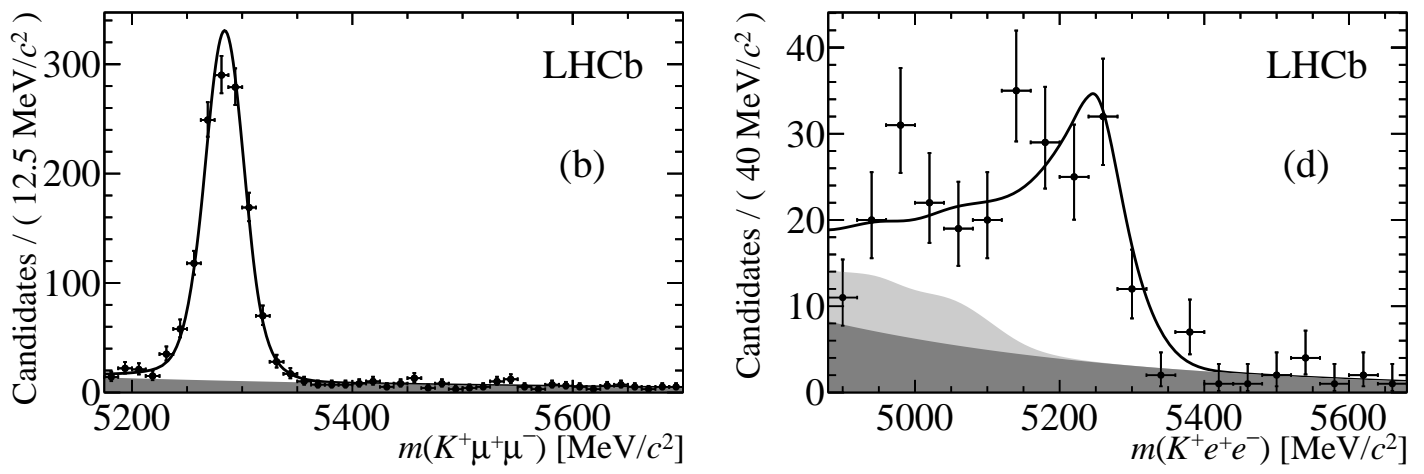

Figure 4: Reconstructed $B^{+}$candidate mass distributions with muons (left) and electrons (right).

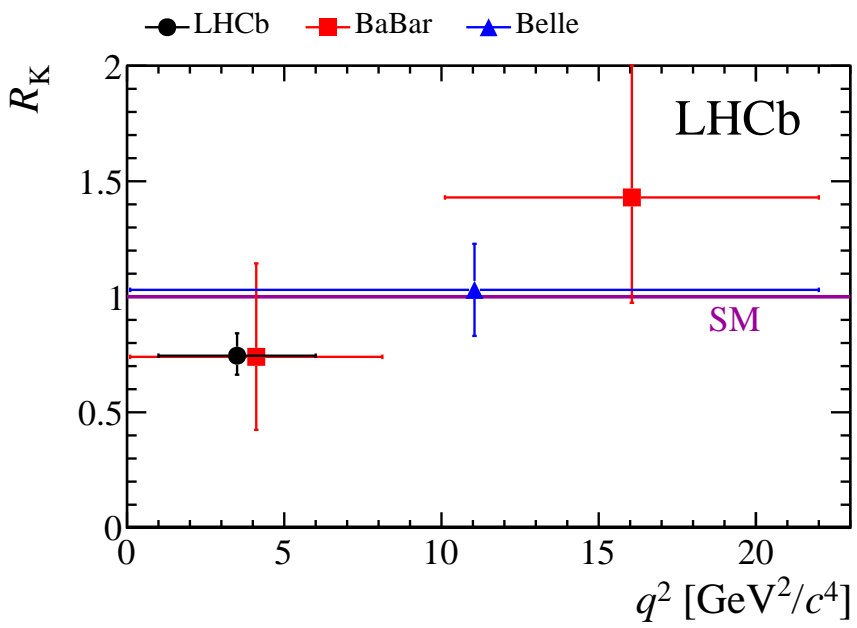

Figure 5: The LHCb measurement of lepton universality in $B^{+} \rightarrow K^{+} \ell^{+} \ell^{-}$decays, $R_{K}$, compared to other experiments.

\section{CKM matrix parameters}

Quark mixing in the SM is described by the $3 \times 3$ unitary CKM matrix which dictates the coupling strength between different flavours of quarks. The CKM elements demonstrate a hierachy across quark generations, with elements further from the diagonal having weaker couplings. Measuring the values of the CKM parameters is an important test of the SM and can provide evidence of new physics. The requirement that the CKM matrix is unitary imposese 6 conditions that the offdiagonal elements of the matrix $V V^{\dagger}=0$. These conditions can be represented as triangles known as the "unitary triangles". Those of particular interest are the $B^{0}$ unitarity triangle which is a representation of the condition, $V_{u d} V_{u b}^{*}+V_{c d} V_{c b}^{*}+V_{t d} V_{t b}^{*}=0$, and the $B_{s}^{0}$ triangle, $V_{u s} V_{u b}^{*}+V_{c s} V_{c b}^{*}+V_{t s} V_{t b}^{*}=0$, shown in Fig. 6.

\subsection{Measuring angle $\beta$}

The unitarity triangle angle $\beta$ can be accessed by measuring the interference between mixing 

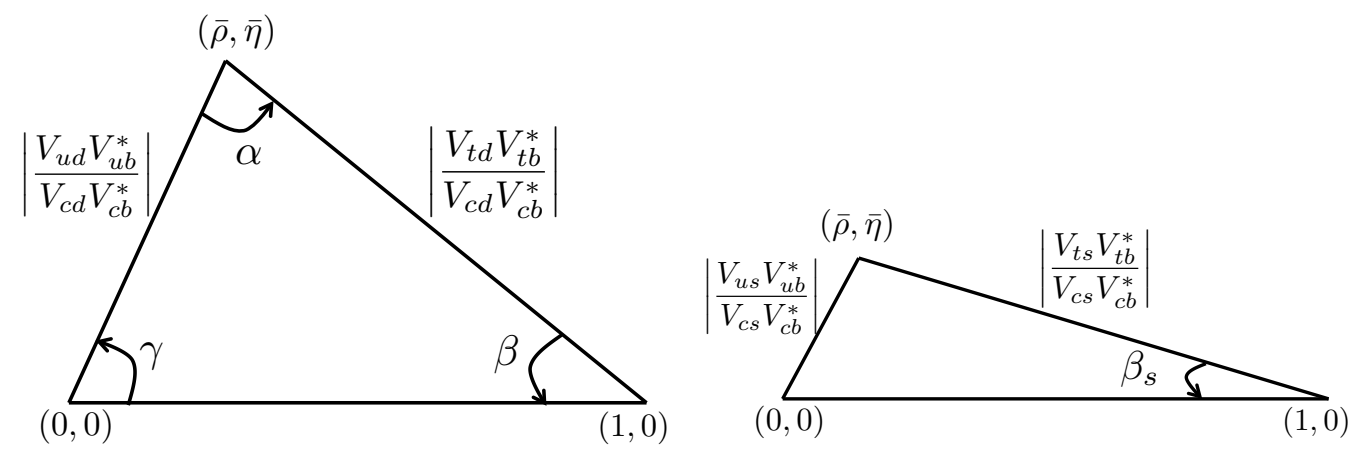

Figure 6: The $B^{0}$ (left) and $B_{s}^{0}$ (right) unitarity triangles.
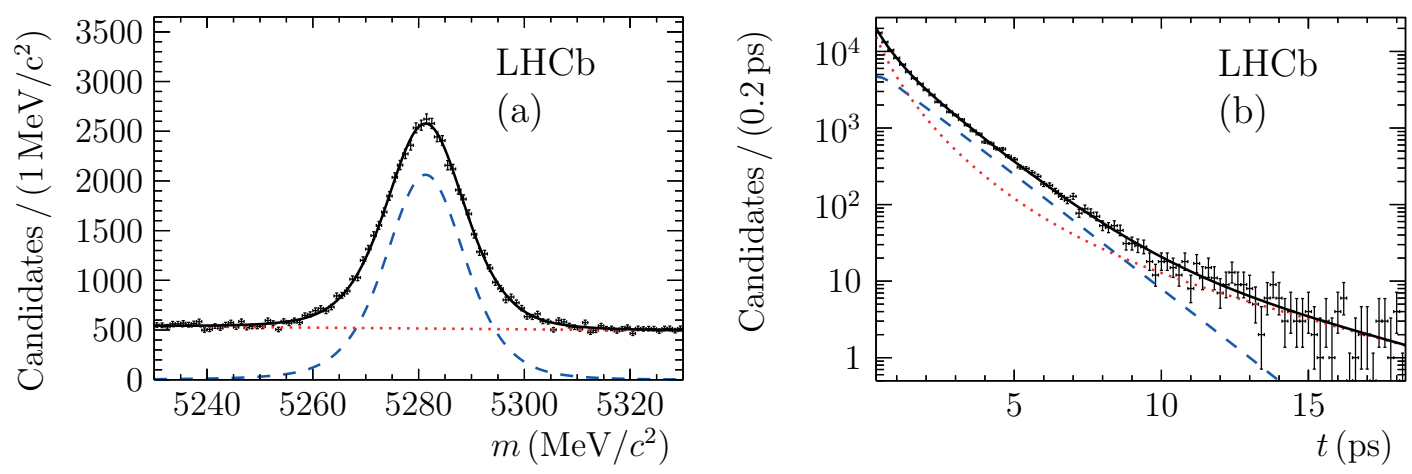

Figure 7: Distribution of the reconstructed mass (left) and decay time (right) of the tagged $B_{s}^{0} \rightarrow J / \psi K_{\mathrm{S}}^{0}$ candidates. The fit projections are shown for the signal (dashed blue), background (dashed red) and total (solid black).

and decay of $B^{0}$ meson decays. The $B^{0} \rightarrow J / \psi K_{\mathrm{S}}^{0}$ decay can occur directly with a phase, $\phi_{D}$, or via mixing to $\bar{B}^{0}$ with a phase $\phi_{M}$ and then decay with phase $-\phi_{D}$. Interference between these gives rise to a $\mathrm{CP}$ violating phase $\phi=2 \beta=\phi_{M}-2 \phi_{D}$. Measuring the decay asymmetry as a function of the decay time gives access to $\sin (2 \beta)$. LHCb has recently updated the measurement of this channel with $3 \mathrm{fb}^{-1}$ [15]. The invariant mass and decay time distributions are shown in Fig. 7. The time-dependent signal yield asymmetry is shown Fig. 8. The LHCb measurement of $\sin (2 \beta)=0.731 \pm 0.035$ (stat) \pm 0.020 (syst) is consistent with the SM prediction and the sensitivity is approaching that of the world average, $\sin (2 \beta)=0.68 \pm 0.02$. Further statistics from LHCb and the Belle II experiment [?] will constrain this measurement further in the near future.

\subsection{Measuring angle $\gamma$}

The angle $\gamma$ is accessed via tree decays of the type $B^{ \pm} \rightarrow D K^{ \pm}$and $B^{ \pm} \rightarrow D \pi^{ \pm}$, with either a $D^{0}$ or $\bar{D}^{0}$, which lead to the same final state. The interference between the favoured $b \rightarrow c$ transition decays and the suppressed $b \rightarrow u$ transistion decays allows access to the angle $\gamma$ which enters in the amplitude for the supressed decays. The results of many $D$ decay modes which have different methodologies are combined together [16], these are summarised in Table 2.

The nominal LHCb result is a combination of all decays of the type $B^{ \pm} \rightarrow D K^{ \pm}$and gives 


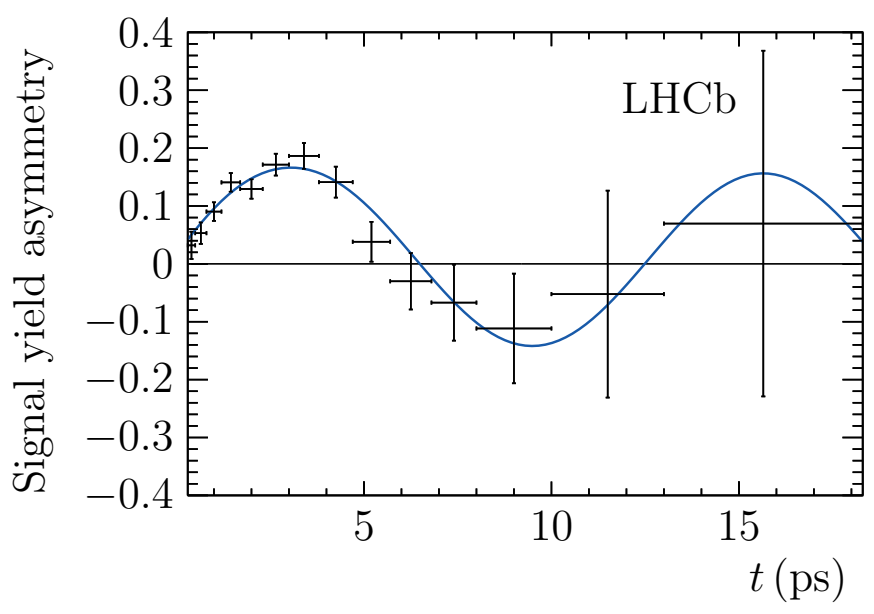

Figure 8: The time-dependent signal asymmetry $\left(N_{\bar{B}^{0}}-N_{B^{0}}\right) /\left(N_{\bar{B}^{0}}+N_{B^{0}}\right)$.

Table 2: Description of the methods and decay channels exploited for the LHCb $\gamma$ combination.

\begin{tabular}{lll}
\hline Method & Decay & Reference \\
\hline \hline GGSZ & $D \rightarrow K_{\mathrm{s}}^{0} \pi^{ \pm} \pi^{\mp}, D \rightarrow K_{\mathrm{s}}^{0} K^{ \pm} K^{\mp}$ & {$[17]$} \\
K3 $\pi$ & $D \rightarrow \pi^{ \pm} K^{\mp} \pi^{ \pm} \pi^{\mp}, D \rightarrow K^{ \pm} \pi^{\mp} \pi^{ \pm} \pi^{\mp}$ & {$[18]$} \\
ADS & $D \rightarrow \pi^{ \pm} K^{\mp}$ & {$[18]$} \\
GLW & $D \rightarrow K^{ \pm} K^{\mp}, D \rightarrow \pi^{ \pm} \pi^{\mp}$ & {$[18]$} \\
\hline
\end{tabular}

$\gamma=\left(72.9_{-9.9}^{+9.2}\right)^{\circ}[16]$. Many Run 1 modes are still to be published with $3 \mathrm{fb}^{-1}$ and continued improvement is expected with the larger statistics of Run 2 and beyond. The expected uncertainty after Run 1 will be near $3^{\circ}$ precision, which is the current indirect precision. The confidence level of the LHCb combined $\gamma$ measurement is shown in Fig. 9. A summary of the current status of unitarity triangle measurements provided by the CKM fitter group [19] is shown in Fig. 10. These are consistent with the SM prediction of 0.07 .

\subsection{CP violation in the $B_{s}^{0}$ system}

Interference between decay and mixing also occurs in the $B_{s}^{0}$ system and gives rise to a $\mathrm{CP}$ violating phase $\phi_{s}$ which in the SM is equivalent to the $B_{s}^{0}$ unitarity triangle parameter $-2 \beta_{s}$. This is very sensitive to new physics as it is small and precisely predicted by the SM. Early Tevatron results of this using the $B_{s}^{0} \rightarrow J / \psi \phi$ decay were tantalising [20, 21]. LHCb (and also CMS and ATLAS [22, 23]) have now clarified this picture with an analysis of $B_{s}^{0} \rightarrow J / \psi K^{+} K^{-}$and $B_{s}^{0} \rightarrow$ $J / \psi \pi^{+} \pi^{-}$decays with $\sim 20$ times the precision of the Tevatron. LHCb have recently published an analysis measuring the time-dependent $\mathrm{CP}$ asymmetry in $B_{s}^{0} \rightarrow J / \psi K^{+} K^{-}$decays with the full Run 1 dataset. This allows measurement of the phase, $\phi_{s}$, as well as the decay widths of the light and heavy mass eigenstates of the $B_{s}^{0} \bar{B}_{s}^{0}$ system. The angular decay distributions and decay time distribution for the decay are shown in Fig. 11. The measured value is $\phi_{S}=-0.058 \pm 0.049 \pm$ $0.006 \mathrm{rad}$ which dominates the world average value of $\phi_{s}=-0.015 \pm 0.035 \mathrm{rad}$. 


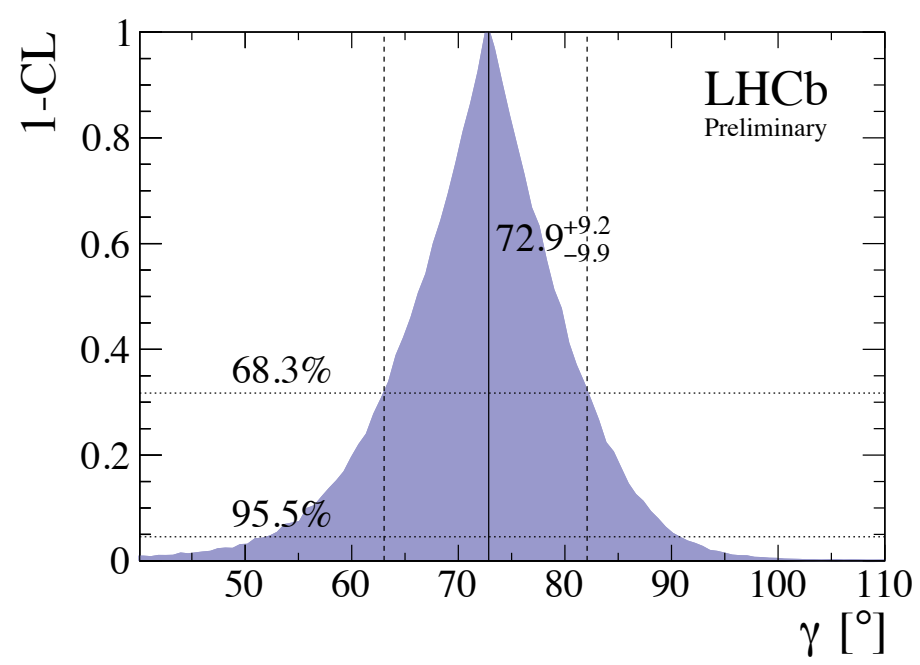

Figure 9: $\mathrm{LHCb}$ combined measurement of $\gamma$ using $B^{ \pm} \rightarrow D K^{ \pm}$decays.

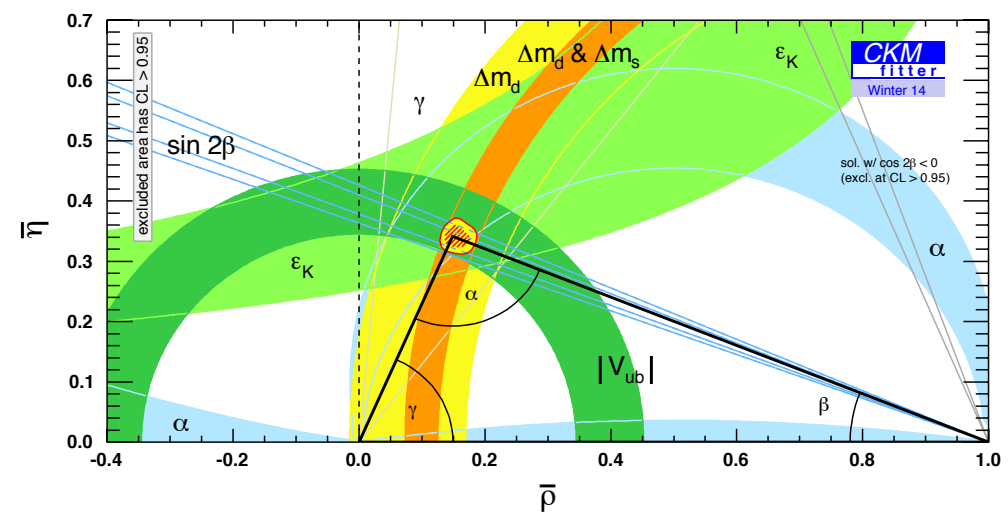

Figure 10: World average of $B^{0}$ unitarity triangle measurements.

\section{Future prospects}

The LHC will start running again and recording physics with $p$ - $p$ collisions at $\sqrt{s}=13 \mathrm{TeV}$ in 2015. The total $b \bar{b}$ cross section will increase by a factor of 1.6. It is expected that $\mathrm{LHCb}$ will collect an additional 6-8 $\mathrm{fb}^{-1}$ of collision data during the whole run. Considerable improvements to the trigger system will facilitate new challenges including performing calibration and alignment of the detector inside the software trigger which will reduce uncertainties arising from differences between the reconstruction in the trigger and offline. Improved precision is expected for many measurements and full exploitation of the Run 1 data is still ongoing.

There are huge improvements planned for 2019 and beyond with the LHCb upgrade. The operational instananeous luminsoity will increase by a factor of 5-10 with the aim of collecting a total of $50 \mathrm{fb}^{-1}$ in Run III. All the subsystems of the LHCb detector will be upgraded and the hardware requirements of the trigger system, and the corresponding systematics uncertainties, 

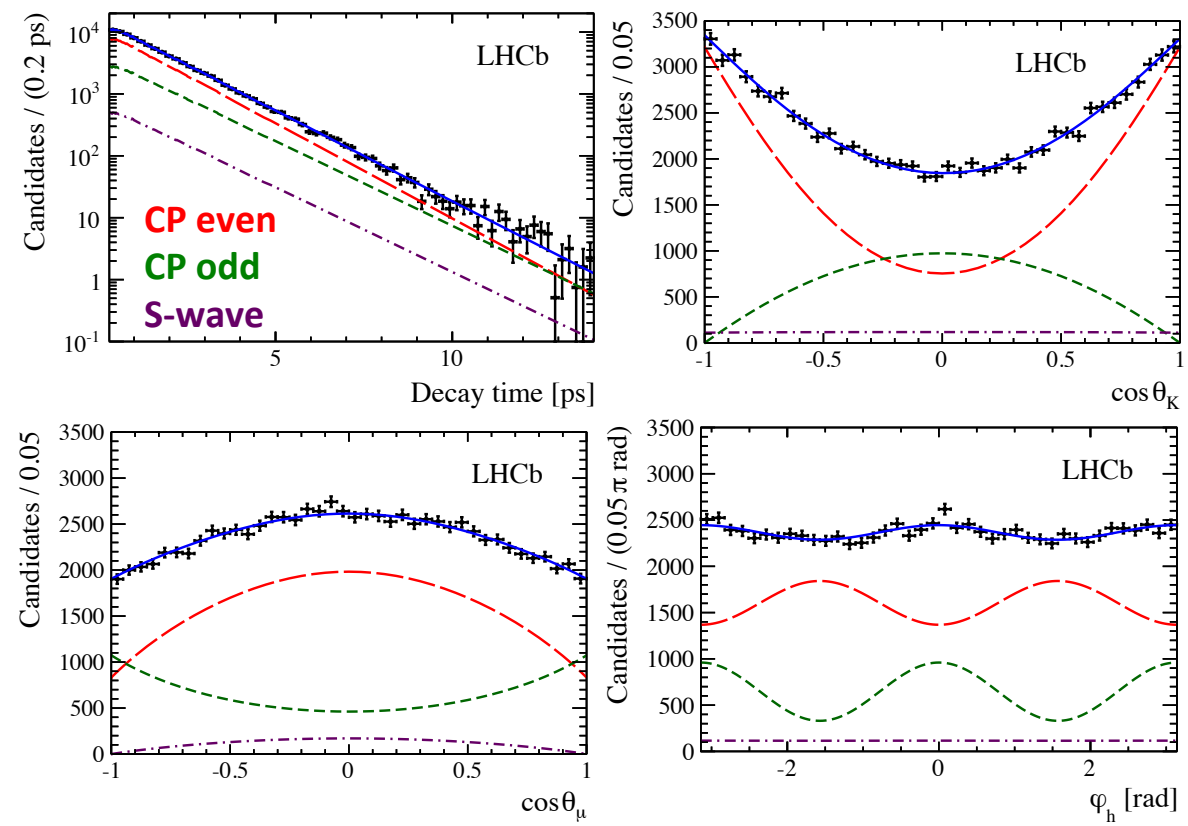

Figure 11: The distributions in data and the fit projections for the CP-even (red), CP-odd (green) and S-wave (purple) components of the decay angle distributions and the decay time.

Table 3: Expected precision for key measurements at LHCb for milestones at the end of Run I, II and III.

\begin{tabular}{|c|c|c|c|c|c|}
\hline Type & Observable & $\begin{array}{c}\text { Current } \\
\text { precision }\end{array}$ & $\begin{array}{l}\text { LHCb } \\
2018\end{array}$ & $\begin{array}{l}\text { Upgrade } \\
\left(50 \mathrm{fb}^{-1}\right)\end{array}$ & $\begin{array}{c}\text { Theory } \\
\text { uncertainty }\end{array}$ \\
\hline \multirow[t]{3}{*}{$B_{S}^{0}$ mixing } & $2 \beta_{S}\left(B_{S}^{0} \rightarrow J / \psi \phi\right)$ & 0.035 & 0.025 & 0.008 & $\sim 0.003$ \\
\hline & $2 \beta_{S}\left(B_{S}^{0} \stackrel{S}{\rightarrow} J / \psi f_{0}(980)\right)$ & 0.17 & 0.045 & 0.014 & $\sim 0.01$ \\
\hline & $A_{\mathrm{fs}}\left(B_{\mathrm{S}}^{0}\right)$ & $6.4 \times 10^{-3}$ & $0.6 \times 10^{-3}$ & $0.2 \times 10^{-3}$ & $0.03 \times 10^{-3}$ \\
\hline Gluonic & $2 \beta_{S}^{\mathrm{eff}}\left(B_{S}^{0} \rightarrow \phi \phi\right)$ & - & 0.17 & 0.03 & 0.02 \\
\hline \multirow{2}{*}{ penguin } & $2 \beta_{S}^{\mathrm{eff}}\left(B_{S}^{0} \rightarrow K^{* 0} \bar{K}^{* 0}\right)$ & - & 0.13 & 0.02 & $<0.02$ \\
\hline & $2 \beta^{\mathrm{eff}^{5}}\left(B^{0} \rightarrow \phi K_{S}^{0}\right)$ & 0.17 & 0.30 & 0.05 & 0.02 \\
\hline \multirow{2}{*}{$\begin{array}{l}\text { Right-handed } \\
\text { currents }\end{array}$} & $2 \beta_{s}^{\text {eff }}\left(B_{S}^{0} \rightarrow \phi \gamma\right)$ & - & 0.09 & 0.02 & $<0.01$ \\
\hline & $\tau^{\mathrm{eff}}\left(B_{S}^{0} \rightarrow \phi \gamma\right) / \tau_{B_{S}^{0}}$ & - & $5 \%$ & $1 \%$ & $0.2 \%$ \\
\hline \multirow{4}{*}{$\begin{array}{l}\text { Electroweak } \\
\text { penguin }\end{array}$} & $S_{3}\left(B^{0} \rightarrow K^{* 0} \mu^{+} \mu^{-} ; 1<q^{2}<6 \mathrm{GeV}^{2} / c^{4}\right)$ & 0.08 & 0.025 & 0.008 & 0.02 \\
\hline & $s_{0} A_{\mathrm{FB}}\left(B^{0} \rightarrow K^{* 0} \mu^{+} \mu^{-}\right)$ & $25 \%$ & $6 \%$ & $2 \%$ & $7 \%$ \\
\hline & $A_{\mathrm{I}}\left(K \mu^{+} \mu^{-} ; 1<q^{2}<6 \mathrm{GeV}^{2} / c^{4}\right)$ & 0.25 & 0.08 & 0.025 & $\sim 0.02$ \\
\hline & $\mathcal{B}\left(B^{+} \rightarrow \pi^{+} \mu^{+} \mu^{-}\right) / \mathcal{B}\left(B^{+} \rightarrow K^{+} \mu^{+} \mu^{-}\right)$ & $25 \%$ & $8 \%$ & $2.5 \%$ & $\sim 10 \%$ \\
\hline Higgs & $\mathcal{B}\left(B_{S}^{0} \rightarrow \mu^{+} \mu^{-}\right)$ & $1.5 \times 10^{-9}$ & $0.5 \times 10^{-9}$ & $0.15 \times 10^{-9}$ & $0.3 \times 10^{-9}$ \\
\hline penguin & $\mathcal{B}\left(B^{0} \rightarrow \mu^{+} \mu^{-}\right) / \mathcal{B}\left(B_{S}^{0} \rightarrow \mu^{+} \mu^{-}\right)$ & - & $\sim 100 \%$ & $\sim 35 \%$ & $\sim 5 \%$ \\
\hline Unitarity & $\gamma\left(B \rightarrow D^{(*)} K^{(*)}\right)$ & $\sim 10-12^{\circ}$ & $4^{\circ}$ & $0.9^{\circ}$ & negligible \\
\hline triangle & $\gamma\left(B_{S}^{0} \rightarrow D_{S} K\right)$ & - & $11^{\circ}$ & $2.0^{\circ}$ & negligible \\
\hline angles & $\beta\left(B^{0} \rightarrow J / \psi K_{S}^{0}\right)$ & $0.8^{\circ}$ & $0.6^{\circ}$ & $0.2^{\circ}$ & negligible \\
\hline Charm & $A_{\Gamma}$ & $2.3 \times 10^{-3}$ & $0.40 \times 10^{-3}$ & $0.07 \times 10^{-3}$ & - \\
\hline$C P$ violation & $\Delta A_{C P}$ & $2.1 \times 10^{-3}$ & $0.65 \times 10^{-3}$ & $0.12 \times 10^{-3}$ & - \\
\hline
\end{tabular}

will be removed entirely. A summary of the expected improvements for many of the important measurements at LHCb is given in Table 3. 


\section{References}

[1] LHCb collaboration, JINST 3, S08005 (2008).

[2] M. Adinolfi, et al., Eur. Phys. J. C73, 2431 (2012).

[3] LHCb collaboration, JINST 8, P02022 (2012).

[4] R. Aaij, et al., JINST 8, P04022 (2013).

[5] L. Breiman, J. H. Friedman, R. A. Olshen, C. J. Stone, Classification and regression trees (Wadsworth international group, Belmont, California, USA, 1984).

[6] Y. Freund, R. E. Schapire, Jour. Comp. and Syst. Sc. 55, 119 (1997).

[7] LHCb collaboration, Phys. Rev. Lett. 110, 021801 (2013).

[8] LHCb collaboration, Phys. Rev. Lett. 111, 101805 (2013).

[9] CMS collaboration, Phys. Rev. Lett. 111, 101804 (2013).

[10] CMS and LHCb collaborations, Submitted to Nature (2014).

[11] D. M. Straub, Nuovo Cim. C035N1, 249 (2012).

[12] F. Mahmoudi, S. Neshatpour, J. Virto, Eur.Phys.J. C74, 2927 (2014).

[13] LHCb collaboration, LHCb-CONF-2015-002 .

[14] S. Descotes-Genon, L. Hofer, J. Matias, J. Virto, JHEP 1412, 125 (2014).

[15] LHCb collaboration, LHCb-PAPER-2015-004 (2015).

[16] T. M. Karbach, Proceedings of the 8th International Workshop on the CKM Unitarity Triangle (2014).

[17] LHCb collaboration, JHEP 1410, 97 (2014).

[18] LHCb collaboration, Phys.Lett. B712, 203 (2012).

[19] J. Charles, et al., LPT-Orsay-15-04 (2015).

[20] T. Aaltonen, et al., Phys.Rev.Lett. 109, 171802 (2012).

[21] V. M. Abazov, et al., Phys.Rev. D85, 032006 (2012).

[22] CMS collaboration, CMS-PAS-BPH-13-012 (2014).

[23] A. collaboration, JHEP 1212, 072 (2012). 